\title{
Kernos
}

Revue internationale et pluridisciplinaire de religion grecque antique

20 | 2007

Varia

\section{SOURVINOU-INWOOD Christiane, Hylas, the Nymphs, Dionysos and Others. Myth, Ritual, Ethnicity}

\section{Sébastien Dalmon}

\section{OpenEdition \\ Journals}

\section{Édition électronique}

URL : https://journals.openedition.org/kernos/366

DOI : $10.4000 /$ kernos.366

ISSN : 2034-7871

\section{Éditeur}

Centre international d'étude de la religion grecque antique

\section{Édition imprimée}

Date de publication : 1 janvier 2007

Pagination : 432-436

ISSN : 0776-3824

\section{Référence électronique}

Sébastien Dalmon, « sourvinou-Inwood Christiane, Hylas, the Nymphs, Dionysos and Others. Myth, Ritual, Ethnicity », Kernos [En ligne], 20 | 2007, mis en ligne le 25 mai 2011, consulté le 08 septembre 2022. URL : http://journals.openedition.org/kernos/366 ; DOI : https://doi.org/10.4000/kernos.366 
que le philosophe déploie devant ses juges, apparemment de manière à provoquer sa condamnation. L'auteur s'oppose aux interprètes qui considèrent la megalêgoria socratique comme un moyen de "suicide volontaire ». Après une étude de l'usage de ce terme dans l'ensemble du corpus de Xénophon, il affirme que celui-ci peut revêtir pour la plupart un sens négatif, celui de la «vantardise » choquante. Or Xénophon accepte que «mettre en avant ses propres mérites » est l'attitude la plus appropriée qui s'impose à des gens en principe modestes, dans des cas particuliers. La défense de Socrate en est une, pense l'A. Étant empêché par le daimonion de préparer un logos rhétorique habituel dans de telles conditions, Socrate se voit poussé à une megalêgoria qui n'est, en fait que la présentation de son ergon philosophique tel quel. Le philosophe reste égal à lui même, suivant l'incitation silencieuse du "signe divin», sans avoir recours à des artifices rhétoriques. En fait, Xénophon semble avoir voulu «corriger» par son propre texte des impressions qu'il a estimées inadmissibles, données par l'Apologie de Platon.

Aldo Brancacci, «The Double Daimôn in Euclides the Socratic» (p. 143-154), sort entièrement de l'étude des textes abordés par les auteurs précédents, en offrant une interprétation inattendue et raffinée de quelques fragments d'Euclide, auteur de dialogues socratiques considérés par le Stoicien Panétius comme douteux. En faisant le lien avec les doctrines socratiques concernant les daimones et le daimonion, telles qu'on peut les déduire par les textes de Platon et de Xénophon, l'auteur avance diverses hypothèses, concernant, d'une part, l'identité du «double daimôn » qu'Euclide croyait assigné à chaque personne et, d'autre part, l'interprétation d'un fragment d'Euclide où se présente la description d'un "autre daimôn ». Les ressemblances constatées marquent des interactions importantes parmi les disciples de Socrate désireux de s'adonner à une écriture « consolatrice » après la perte du maître.

L'ouvrage contient encore une bibliographie des sources et des notes biographiques sur les auteurs. Enfin, une série d'index constituent des outils précieux pour guider les lecteurs vers les intérêts plus pointus dans cet ensemble de commentaires abondants sur les divers textes où figure l'intriguant daimonion sêmeion de Socrate, finalement aussi atopon que le philosophe lui-même.

Aikaterini Lefka

(Université de Liège, Université de Luxembourg, Towson University)

Sourvinou-Inwood Christiane, Hylas, the Nymphs, Dionysos and Others. Myth, Ritual, Ethnicity, Stockholm, Åströms Förlag, 2005. 1 vol. 16,5 × 24 cm, 421 p. (Acta Instituti Atheniensis Regni Sueciae, Series in-8²,19). ISBN : 91-7916-051-4.

Le titre du dernier livre de Chr. Sourvinou-Inwood (C.S.-I.) laisse songeur: on comprend la relation d'Hylas, le premier nommé, avec les Nymphes qui l'ont enlevé, mais que vient faire ici Dionysos qui n'apparait pas dans sa légende? Et qui sont ces mystérieux « autres » qui suivent? Le sous-titre lève un peu le voile en nous indiquant qu'il ne s'agit pas ici de traiter seulement du célèbre mythe (bien connu par l'iconographie, jusqu'au célèbre tableau de Waterhouse daté de 1896 et conservé au Manchester's City Art Gallery), mais aussi du rituel (dont il représente l'étiologie, dans la cité grecque de Kios en Asie Mineure) et de l'« ethnicité » (ces mythe et rituel articulant une représentation de l'identité hellénique en terre mysienne). L'objet du livre est en effet de reconstituer le "nexus mythico-rituel» d'Hylas à Kios, à travers une investigation précise des sources. L'A. pratique une analyse structurale, en comparant ces sources à d'autres discours où l'on retrouve les mêmes thématiques : interactions culturelles et religieuses entre Grecs et non-Grecs, récits coloniaux de fondation, représentation de l'identité de sa communauté (ce qu’elle nomme «ethnicité»), rapprochement avec les mythes et cultes d'autres divinités (dont Dionysos), nature et fonc- 
tions des Nymphes, festivals d'arrivée d'une divinité, catégories problématiques de certains destinataires du culte, etc.

Le $1^{\text {er }}$ chapitre de la $1^{\text {re }}$ partie (Greek religion and its hinterland; methodologies and the (post modern) wilderness) est en fait une introduction présentant la méthodologie de C.S.-I. pour l'étude de la religion grecque. Elle souligne son aspect essentiellement rituel, et donc la nécessité de s'intéresser de près aux actes du culte. Elle s'inscrit dans la lignée de Nilsson en affirmant qu'il ne faut pas hésiter à reconstituer ceux pour lesquels on ne dispose que de sources fragmentaires. Elle critique au reste le «scepticisme» qui lui semble surévalué dans les actuelles études classiques. Mais les tentatives de reconstitution ne se valent pas toutes, et elle a raison de souligner à cet égard qu'une nécessaire prudence s'impose, afin de minimiser l'impact de nos propres représentations culturelles contemporaines. De plus, les significations d'un texte ou d'une image peuvent être polysémiques, ambiguës et multivoques... Les mythes doivent être comparés à d'autres, car ils sont structurés par des «schémas» (les « mythèmes » de Lévi-Strauss), eux-mêmes structurés par les réalités, croyances et idéologies de la société qui les produit. Les différentes versions du mythe d'Hylas utilisent ainsi plusieurs schémas qui interagissent entre eux: "fondation d'une cité ", "enlèvement érotique »... Localisé à Kios de Mysie, ce mythe est indéniablement local, mais renvoie également à des mythes panhelléniques (Héraclès, les Argonautes). Il ne nous est guère connu que par des sources hellénistiques, même si l'on peut supposer qu'il est plus ancien (Eschyle et Aristophane semblent y faire allusion).

Le $2^{\text {e }}$ chapitre de la $1^{\text {re }}$ partie s'attache à la notion d'« ethnicité » et à la définition de l'hellénisme (Greekness). Les représentations qu'ont les communautés grecques d'elles-mêmes et des autres peuples font l'objet d'analyses qui courent tout au long du livre, de même que les interactions culturelles entre Grecs et non-Grecs. La difficulté est qu'il existe plusieurs types d'« identités ethniques » dans le monde grec antique : la distinction entre Hellènes et Barbares ne saurait faire oublier le partage des premiers entre Ioniens, Doriens, Éoliens et Achéens. Les ancestralités mythologiques viennent compliquer le problème, même si C.S.-I. critique quelque peu à cet égard les analyses de J. Hall (Ethnic identity in Greek antiquity, Cambridge, 1997). Pour elle, tous ceux qui se percevaient eux-mêmes comme Grecs l'étaient, malgré d'éventuels apports généalogiques barbares. L'ethnicité grecque ne saurait ainsi se définir par l'ancestralité. Pour autant, la perception des non-Grecs existait selon elle bien avant les guerres Médiques, dès l'Iliade, malgré la communauté de culture qui semble unir les Troyens à leurs ennemis (qui ne sont pas encore nommés « Hellènes »).

La $2^{\text {e }}$ partie est centrée sur les sources. Sont d'abord présentées les données littéraires sur le mythe et le culte d'Hylas. L'histoire du jeune compagnon d'Héraclès, présente dans plusieurs textes fragmentaires (Hellanikos, Socrate d'Argos, ou Antikleides d'Athènes qui le confond avec Hyllos) et, de manière allusive, dès l'époque classique (Aristophane, Ploutos, 1127; Eschyle, Perses, 1055), est surtout narrée dans les Argonautiques d'Apollonios de Rhodes (I, 1207-1357), la 13e Idylle de Théocrite, et les Métamorphoses de Nicandre résumées dans l'œuvre homonyme d'Antoninus Liberalis (chapitre 26). Elle donne lieu à quelques variantes: par exemple, c'est tantôt une seule Nymphe qui enlève Hylas (Apollonios), tantôt plusieurs (Théocrite, Nicandre). Le rituel effectué chaque année par les habitants de Kios en l'honneur d'Hylas (lamentation, sacrifice près d'une source, invocation et même course rituelle) est évoqué par Strabon (XII, 4, 3) qui parle d'un festival et d'une oreibasia dans le mont Arganthonion. Hésychios précise que la lamentation rituelle est effectuée par des femmes mysiennes. Le Romain Solin localise quant à lui le rituel près d'un lac. Les sources sont aussi numismatiques (des monnaies de Kios représentent Hylas allant chercher de l'eau) et iconographiques. C.S.-I. pense ainsi reconnaitre une représentation du rituel célébré pour Hylas sur une coupe athénienne à figures rouges du ve s. av. J.-C. attribuée au peintre de 
Télèphe (reproduite p. 88). Son analyse, méthodique et précise, emporte la conviction, même s'il s'agit avant tout d'une interprétation. Elle n'évoque pas, en revanche, les sources iconographiques plus tardives qui ne sont pas en lien avec Kios (ainsi les mosaïques romaines représentant Hylas et les Nymphes, comme le très bel exemple conservé au musée archéologique de Saint-Romain-en-Gal).

Les Nymphes font l'objet de la 3 e partie, et d'une analyse qui dépasse celle du mythe d'Hylas. L'A. s'intéresse d'abord à la catégorie même de Nymphes, qui se subdivise en plusieurs sous-groupes liés à leur habitat (Oréades des montagnes, Hydriades et Naïades des sources et des fleuves, Dryades et Meliai des arbres...). Elle évoque aussi les Océanides et les Néréides liées à la mer (ce n'est guère vrai pour le premier groupe, qui préside surtout aux sources souterraines). Certaines Nymphes ont une personnalité individuelle bien précise (Calypso, Aréthuse...), mais elles sont beaucoup plus nombreuses à être perçues en termes de collectivité (l'une des modalités du «féminin pluriel» mis en évidence par Nicole Loraux). Puissances généralement bienveillantes envers les hommes, elles ont des liens avec la sphère du mariage, ne serait-ce que par leur nom, qui sert aussi à qualifier des "fiancées » ou « jeunes mariées » humaines, en l'occurrence un statut intermédiaire entre la parthénos (ou la korè) et la gunè (ou la mèter). Certaines sources les situent aussi dans un statut intermédiaire entre hommes et dieux : en effet, elles restent toujours de jeunes déesses (théar), vivent très longtemps, mais ne sont pas immortelles, bien que se nourrissant d'aliments divins. La catégorie n'a pas des contours bien définis. Il est parfois difficile de distinguer Nymphe et déesse (Océanides, Néréides), mais aussi Nymphe et héroïne (Kallisto) ou jeune mortelle (la polysémie du mot n'arrange pas les choses). Les Nymphes sont des kourotrophes, comme celles de Nysa (ou les Hyades) qui ont élevé Dionysos, et sont associées aux initiations adolescentes qui font passer de la jeunesse à l'âge adulte. Elles peuvent se montrer également très ambiguës en enlevant des jeunes gens (à qui elles peuvent accorder l'immortalité, comme dans le mythe d'Hylas), ou en possédant des mortels. C.S.-I. a raison, à cet égard, de souligner que la nympholepsie semblait être plutôt perçue comme positive, au regard d'autres théolepsies, car elle allait souvent de pair avec l'inspiration prophétique. Les Nymphes sont également souvent liées à des fondations de cités, en tant qu'éponymes ou épouses et mères de héros fondateurs. Cet aspect permet de légitimer par exemple l'installation d'une colonie en l'ancrant dans le paysage local.

La démarche comparative est au centre de la $4 \mathrm{e}$ partie, qui concerne les jeunes hommes mythiques faisant l'objet d'une lamentation rituelle publique. Le rituel de lamentation des femmes de Kios est comparé aux discours mythiques et rituels sur Adonis, Hyakinthos, Hippolyte, Skephros et Linos, mais aussi Maneros, Bormos, Mariandynos et Lityersès. Certaines de ces figures ont d'autres points communs avec Hylas, notamment Adonis qui est lui aussi l'objet de la passion amoureuse d'une déesse. Ce trait se retrouve dans le personnage d'Attis, étudié dans un chapitre spécifique. Le compagnon de la Mère des dieux faisait lui aussi l'objet d'une invocation sur une montagne, mais il n'a pas été immortalisé comme le jeune éromène d'Héraclès (ce dernier étant d'ailleurs lui aussi passé du statut de mortel à celui d'immortel). Le mythe d'Hylas peut avoir été influencé par ces autres figures mythiques, de même que le rituel de Kios a pu l'être par d'autres lamentations cultuelles. Mais C.S.-I. conclut que les influences grecques ont eu peut-être plus d'importance que les anatoliennes.

La $5^{\mathrm{e}}$ partie poursuit les investigations en ce sens en s'attachant à l'étude de la figure de Dionysos, en relation avec ses festivals d'arrivée. Ce type de rituel est attesté également pour Korè à la source Kyanè, sur le territoire de Syracuse. C.S.-I. analyse également les rapports entre Dionysos et Apollon à Delphes, remettant en question l'idée que Dionysos était absent du sanctuaire pendant la période où Apollon y était présent. Elle explore les paradoxes de la 
double figure de Dionysos, le fils de Perséphone (tué par les Titans) et le fils de la mortelle Sémélé (qui ne semble pas avoir connu la mort, même s'il s'est réfugié dans la mer lors des agressions de Lycurgue ou de Persée, ou s'il est allé chercher sa mère dans les Enfers). L'A. analyse ensuite les rituels d'arrivée de Dionysos, notamment ceux de Lerne, de Rhodes et de Delphes.

La $6^{\mathrm{e}}$ partie opère un retour sur le mythe d'Hylas. Elle le relie à celui de la fondation de Kios, dont le ketistes est Héraclès ou Polyphème, les compagnons du jeune homme. Le fait que le mythe implique aussi des Nymphes, souvent éponymes de cités dans d'autres légendes, inscrit la figure d'Hylas dans le paysage local. L'oikistès historique, souvent présent dans les récits à la suite du fondateur mythologique ayant vécu à l'âge hérö̈que (comme à Cyrène ou Tarente), est dans le cas de Kios une figure assez inconsistante, malgré son éponymie. C.S.-I. relie également le mythe d'Hylas aux rites d'initiation adolescents (impliquant généralement une mort symbolique), le comparant à d'autres récits où la dimension homoérotique est présente (Ganymède éromène de Zeus, comme Hylas d'Héraclès). Elle analyse également le thème de l'enlèvement érotique par une divinité, qu'il soit hétérosexuel (Hylas, Tithon, Phaéton) ou homosexuel (Ganymède), souvent lié à des mythes d'immortalisation. Elle conclut de ces analyses que le mythe d'Hylas est un véritable mythe de fondation pour Kios, d'autant plus que le schéma «fondation d'une cité » implique parfois l'idée d'un jeune homme trop tôt disparu (un éphèbe ayant échoué) qui devient éponyme. Dans le cas d'Hylas, le jeune Grec enlevé par une (ou des) Nymphe(s) devient une divinité liée au paysage, enracinant symboliquement la colonie dans ce dernier, et justifiant de ce fait la colonisation. On a cependant ici une inversion du schéma plus habituel d'une Nymphe locale s'unissant à un dieu grec pour donner naissance au héros fondateur. Le mythe d'Hylas est ensuite comparé aux mythes de fondation de la métropole de Kios, Milet, qui ont pu l'influencer. Cependant, l'accent, dans ce cas là, est moins mis sur la fondation héroïque par des Crétois (impliquant des schémas homoérotiques, comme la relation entre Sarpédon et Milétos, ou la présence de Nymphes, dans les histoires de Kaunos et Byblis) que sur la fondation historique ionienne par Nélée, fils de Kodros.

La 7e partie revient sur le rituel d'Hylas à Kios. L'étude de la lamentation et du sacrifice (de type thusia) sont l'occasion d'une comparaison avec des rituels semblables impliquant d'autres héros ou mortels divinisés, comme Ino-Leucothéa, Molpadia-Hémithéa, DictynnaBritomartis, Glaukos (qui annonce au reste aux Argonautes le sort d'Hylas), Amphiaraos ou Trophonios, sans parler des Hyakinthides d'Athènes. La recherche rituelle d'Hylas est rapprochée de celle de Dionysos aux Agrionies de Chéronée ou de Korè dans les Mystères d'Eleusis. La triple invocation d'Hylas par le prêtre (auquel répond l'écho, censé être une manifestation épiphanique) évoque selon l'A. un festival d'arrivée divine, qui a pu comporter aussi une course autour de la source. Nous ne savons pas en fait si ce rituel autour d'Hylas faisait partie d'un festival plus large, au cours duquel, par exemple, les Nymphes ou Héraclès auraient pu également recevoir un culte. C.S.-I. en conclut néanmoins qu'une commémoration annuelle de la fondation de Kios devait être centrée sur Hylas. La probable connexion de ce dernier avec les initiations adolescentes permettait également, selon elle, d'intégrer les hommes les plus jeunes à cette cérémonie civique, et donc à la communauté des citoyens. Le lien est également souligné entre la cité et les éléments naturels de son territoire (montagne, fleuve homonyme, source ou lac), ce que renforce la signification probable du nom d'Hylas (dérivé de bylè, la forêt).

$\mathrm{La} 8^{\mathrm{e}}$ partie (The construction of a divinity and a colonial discourse: Ethnicity, ideology, religious interaction) est en fait une conclusion générale, rappelant la complexité du discours sur Hylas, construit à travers des interactions entre divers schémas mythico-rituels, incluant également des éléments non-Grecs localisés dans le voisinage de Kios. Il s'agit à la fois d'un mythe de 
fondation (soulignant la continuité de la fondation milésienne avec l'âge héroïque) et d'un mythe d'immortalisation, construisant le lien d'une cité avec une divinité grecque mais enracinée dans un territoire asiatique. La construction d'Hylas comme divinité (probablement dès les origines de la colonisation archaïque) a un aspect éminemment politique et permet de légitimer symboliquement la colonie, tout en soulignant la relation de coopération entre les Grecs et les autochtones Mysiens.

L'étude, menée avec précision et érudition, illustre magistralement la plasticité de la religion grecque, et montre comment certains mythes et rituels sont transformés et adaptés à d'autres contextes. La démonstration est convaincante, même si elle donne lieu à de nombreuses répétitions et s'égare parfois dans des développements qui font quelque peu oublier Hylas (ainsi des longs chapitres sur Dionysos, ou des réflexions sur l'ethnicité). L'A. explore en fait plusieurs pistes, égarant quelquefois un peu son lecteur, mais tous les éléments s'ajustent finalement dans une conclusion brillante qui reprend les différents thèmes abordés dans chaque partie. On peut contester ici ou là certaines analyses. Ainsi, les Océanides, comme les Néréides, sont associées à l'élément marin, et ces deux groupes de déesses sont considérés comme des Nymphes (p. 103), ce qui est très peu attesté dans les sources, surtout pour les filles de Nérée (ces dernières sont des divinités des eaux salées, alors que les Nymphes sont plutôt liées aux eaux douces, comme les Océanides filles du Fleuve primordial Okéanos). L'ensemble forme néanmoins un ouvrage dense et d'une grande qualité. L'étude sur les Nymphes, bien qu'assez brève à l'échelle de l'ouvrage (14 pages) offre ainsi un complément très problématisé au livre de Jennifer Larson (Greek Nymphs. Myth, Cult, Lore, Oxford, 2001). Elle s'offre comme une invitation stimulante à poursuivre les recherches sur ces divinités très particulières du panthéon grec, qui ne se résument pas au rôle de ravisseuses de beaux jeunes hommes allant chercher de l'eau à la source. Après tout, chez Théocrite, Hylas est aussi consolé par ces déesses kourotrophes qui le prennent sur leurs genoux.

Que ce compte rendu, de ce qui est malheureusement le dernier livre de C.S.-I., récemment disparue, soit l'occasion de rendre hommage à cette éminente helléniste qui a ouvert, par ses travaux bien souvent innovants, entre archéologie, philologie, structuralisme, sciences religieuses et études iconographiques, des voies fécondes à la recherche.

Sébastien Dalmon

(Université Paris VII - Denis Diderot)

SFAMENI GASPARro Giulia (éd.), Modi di comunicazione tra il divino e l'umano. Tradizioni profetiche, divinazione, astrologia e magia nel mondo mediterraneo antico. Atti del II Seminario Internazionale Messina 21-22 Marzo 2003, Cosenza, Edizioni L. Giordano, 2005. 1 vol. $14 \times 20,5 \mathrm{~cm}, 383$ p. (Hierá. Collana di studi storicoreligiosi, 7. Temi e problemi della Storia delle Religioni nell'Europa contempranea, 2). ISBN : 88-86919-16-6.

Le volume édité par G. Sfameni Gasparro rassemble les contributions de plusieurs grands spécialistes d'histoire des religions italiens et espagnols réunis lors d'un séminaire international tenu à l'Université de Messine en mars 2003. Les différents articles (pour le détail des différents titres et de la pagination, voir Kernos 19 [2006], p. 517) s'interrogent sur les modes de communication que les hommes du monde méditerranéen antique ont mis en œuvre pour entrer en contact avec leurs dieux. Les diverses traditions prophétiques, les pratiques divinatoires, l'astrologie et la magie sont donc envisagées dans le but de mieux cerner la problématique de la définition de l'identité religieuse de l'Antiquité, qu'elle soit juive, chrétienne ou « païenne». 